\title{
Paediatric Research Society
}

\section{9th Meeting, Royal Hospital for Sick Children, Glasgow 1 and 2 October 1971}

Clicks, honks, and whoops. D. Pickering (Radcliffe Infirmary, Oxford). Three cases are described which were originally diagnosed as having ventricular septal defect, pulmonary stenosis, and innocent praecordial honk. These have been reinvestigated using pixie accelerometer, phonocardiographic techniques (Bew et al., 1971). All three have been shown to have clicks which move with posture, Valsalvas, and other manoeuvres altering the left ventricular end-diastolic volume. These features enable a diagnosis to be made of prolapsing posterior cusp of the mitral valve.

REFERENCE

Bew, F. E., Pickering, D., Sleight, P., and Stott, F. D. (1971). 'Pixie' cardiography. Accelerometer applications to phonocardiography and displacement cardiography in childhood. British Heart fournal, 33, 702.

Measurement of total respiratory resistance using oscillation technique. Jeremy Cogswell (introduced by David Hull) (The Hospital for Sick Children, Great Ormond Street, London). A method for measuring total respiratory resistance by a forced oscillation technique has been described in adults. The technique requires minimal co-operation and causes no discomfort, and therefore can be used in children of all ages.

The details of the technique with modification required for children were described. Values for total respiratory resistance in 170 healthy schoolchildren aged 6-12 years were similar to values of airways resistance using a total body plethysmograph. Reproducible results were obtained in children between the ages of 2 and 5 years.

\section{REFERENCE}

Fisher, A. B., DuBois, A. B., and Hyde, R. W. (1968). Evaluation of the forced oscillation technique for the determination of resistance to breathing. Fournal of Clinical Investigation, 47, 2045.

Paediatric electrodiagnosis. Allie Moosa (Department of Child Health, University of Sheffield). The measurement of the conduction velocity of the peripheral nerve and the application of electromyography (EMG) has proved invaluable in the diagnosis of peripheral neuropathy and in the differentiation of the various forms of muscle weakness in infancy and childhood.

Slow nerve conduction velocities have been found in patients with some of the leucodystrophies, e.g. Krabbe's and metachromatic leucodystrophies, and this has provided a useful screening test for the leucodystrophies in patients with progressive degenerative diseases of the nervous system. Hypothyroid infants have also been found to have slow conduction velocities probably due to retarded myelination. The diagnosis of a peripheral neuropathy due to other causes, e.g. peroneal muscular atrophy, infections, polyneuritis, can also be confirmed by this test.

Needle EMG provides information about the state of the lower motor neurone. Characteristic changes are seen in lesions affecting the anterior horn cell, peripheral nerve, and muscle fibres. The EMG is therefore useful in the differential diagnosis of the floppy infant syndrome and of the child with proximal muscle weakness. It is particularly useful in the diagnosis of dystrophia myotonica and in assessing the prognosis of peripheral nerve lesions, e.g. facial palsy.

The quantitation of the EMG signal has led to its use in detection of carriers of Duchenne dystrophy.

Diagnostic advances in neuromuscular disorders. Victor Dubowitz (Department of Child Health, University of Sheffield). The application of modern histochemical techniques to the assessment of muscle biopsies has led to the recognition of a number of new conditions, such as central core disease and nemaline myopathy, which may masquerade clinically as a dystrophy, and readily be missed with routine histological stains.

'All that waddles is not dystrophy'.

The identification of histochemical fibre types within muscle has also made possible the recognition of selective involvement of fibre types in certain disease situations. Examples include type I atrophy in dystrophia myotonica, type II atrophy in some cases of polymyositis, and fibre type grouping as may be seen in neurogenic atrophy, even in the absence of change in fibre size, thus making possible the diagnosis of these conditions where routine stains show no obvious abnormality.

Cases presented to illustrate these conditions include two floppy infants and their apparently normal related mothers (aunt and niece) with dystrophia myotonica; a mother with central core disease affecting $99 \%$ of the muscle fibres in her biopsy and her minimally affected son with eccentric central cores in $3 \%$ of his type I fibres; a series of children with spinal muscular atrophy of varying severity; two adults with polymyositis and selective type II atrophy and a 4-year-old girl with a 
bizarre 'polymyositis', with bizarre disruptive changes in enzyme distribution in the muscle fibres.

National inquiry into convulsive disorders in childhood. Euan M. Ross (introduced by Dr. Peter M. Dunn) (Department of Child Health, University of Bristol). A detailed study is being made of the incidence, nature, and pattern of convulsive disorders reported in the 15,000 surviving children born in England, Scotland, and Wales during the week 3-9 March 1958, and originally included in the Perinatal Mortality Study. $89 \%$ of these children were traced and studied as the National Child Development Study (1958 cohort).

As a result of comprehensive medical, social, and educational studies made when the children were aged 7 and repeated at age 11 , it was possible to select 1100 children who had been reported as having had one or more convulsions, faints, or breath-holding episodes. From an exhaustive study of individual records it was possible to identify 347 children who fulfilled one or more of the following: (1) History of anticonvulsant medication. (2) Had had an EEG. (3) Regarded by a doctor as being 'epileptic'. (4) Had one or more definite seizures after age 5. (5) Had had three definite seizures at some time. (6) Teacher reported a seizure at school. (7) One or more seizures reported but also attends a special school.

Precoded questionnaires were sent to both the practitioners and consultants and, in certain cases, local authority doctors, who had looked after these children. To date there has been a 95\% reply rate. In 98 children $(6 \cdot 3$ per 1000$)$ a doctor has at some stage regarded the child as being 'epileptic'. Only 32 children however, have had one or more fits since age 8 years ( 2 per 1000 ). Only 2 children appeared to have uncomplicated petit mal though a further 9 had mixed petit and grand mal. Patterns of drug administration were briefly described.

\section{REFERENCE}

Davie, R., Butler, N., and Goldstein, H. (1972). From Birth to Seven Years. Longmans, London.

Substrate induction of galactokinase in cultured fibroblasts from subjects heterozygous and homozygous for galactokinase deficiency. F. Zacchello, P. F. Benson, P. Croll, F. Giannelli, and T. P. Mann (Paediatric Research Unit, Guy's Hospital Medical School, London S.E.1). Skin fibroblasts from a patient with galactokinase deficiency (Cook, Don, and Mann, 1971), the parents, and 4 controls were cultured in minimum essential Eagle's medium containing 10\% human serum, either without or with galactose (5 or $15 \mathrm{mg} / 100 \mathrm{ml}$ culture medium). Fibroblasts were harvested and assayed for galactokinase activity at intervals during a 15-day period, during which time the cells were trypsinized approximately every 7 days and the medium was changed every 3 days.

Mean galactokinase activity for normal fibroblasts was $6 \cdot 18$ units ( $n$ moles of galactose 1-phosphate formed/hr per mg fibroblast protein) without galactose in the medium. In the presence of galactose, activity was induced by the 3 rd day, reached a maximum by the 6 th day, and remained at the induced level until the 15th day. Mean activities were 9.45 units (with galactose $5 \mathrm{mg} / 100 \mathrm{ml}$ ) and 9.97 units (with galactose $15 \mathrm{mg} / 100 \mathrm{ml}$ ).

The patient with galactokinase deficiency had activities of 0.26 and 0.38 units without and with galactose in the medium $(15 \mathrm{mg} / 100 \mathrm{ml})$, respectively. The father had a mean galactokinase activity of $3 \cdot 20$ units $(51.8 \%$ of control) and the mother 2.68 units $(43.4 \%$ of control). Mean activities in the presence of galactose $(15 \mathrm{mg} / 100 \mathrm{ml})$ were: father $4 \cdot 10$ units $(43.8 \%$ of control), mother 3.00 units $(32.0 \%$ of control).

Fibroblast galactokinase thus has properties both of a constitutive enzyme (since it is present in cells cultured in a medium without substrate) and of a substrate inducible enzyme. Galactokinase activity in heterozygous cells was intermediate between that of cells that were homozygous normal and those that were homozygous for galactokinase deficiency. Both in the induced and uninduced states, galactokinase activities were higher in the father $(43.8 \%$ and $51.8 \%$ of control values respectively) than in the mother $(32.0 \%$ and $43.4 \%$ of control values respectively).

\section{REFERENCE}

Cook, J. G. H., Don, N. A., and Mann, T. P. (1971). Hereditary galactokinase deficiency. Archives of Disease in Childhood 46, 465 .

Plasma levels of cystine in homocystinuriaco I. B. Sardharwalla and B. Fowler (Willink BiochemicaE Genetics Laboratory, Royal Manchester Children's Hospital, Pendlebury, Manchester). In homocystinuria plasma levels of cyst(e)ine are low; one explanation for this finding is the lack of methionine conversion to cysteine. It is probable that there is a different and perhaps more significant reason for the reduced concentrations of cyst(e)ine. In this paper results of two studies are presented to expound the latter view.

In the first, a 7-year-old pyridoxine-resistant patient was given (1) low methionine diet followed by (2) high methionine diet (by adding $\mathrm{L}$-methionine to the basic diet) to which (3) betaine hydrochloride was then added. Throughout the study, dietary cystine was kept constant. Measurements of sulphur amino acids in the plasma and urine were performed by ion exchange chromatography, the column effluent being simultaneously monitored with ninhydrin and iodoplatinate analytical systems. The results showed that (1) there was a reciprocal relation between the plasma levels of homocyst(e)ine and cyst(e)ine, and (2) cyst(e)ine was increased in spite of very high levels of methionine during betaine therapy.

In the second study, cysteine loads were given to a 21-year-old pyridoxine-resistant patient (1) before and (2) during betaine therapy while the patient was on a constant normal diet. The result showed that on a fixed normal diet plasma levels of homocysteine-cysteinedisulphide increased significantly and there was a 